\title{
De la verve avant toute chose
}

À propos d'Albert Cohen

\section{Maxime Decout}

\section{(2) OpenEdition \\ Journals}

Édition électronique

URL : https://journals.openedition.org/recherchestravaux/715

DOI : $10.4000 /$ recherchestravaux.715

ISSN : 1969-6434

Éditeur

UGA Éditions/Université Grenoble Alpes

Édition imprimée

Date de publication : 15 décembre 2014

Pagination : 123-134

ISBN : 978-2-84310-291-2

ISSN : 0151-1874

Référence électronique

Maxime Decout, « De la verve avant toute chose », Recherches \& Travaux [En ligne], 85 | 2014, mis en ligne le 15 juin 2016, consulté le 29 octobre 2021. URL : http://journals.openedition.org/ recherchestravaux/715; DOI : https://doi.org/10.4000/recherchestravaux.715 
Maxime Decout

Université Lille 3 - Charles de Gaulle

\section{De la verve avant toute chose À propos d'Albert Cohen}

Si le $\mathrm{Xx}^{\mathrm{e}}$ siècle a connu une crise de la parole, cette crise pourrait aussi être considérée comme une crise de la verve. À l'aune des œuvres de Roussel, Perec, Pinget, Robbe-Grillet, puis Ernaux ou Modiano, c'est en effet une écriture blanche, neutre, plate, en mal de verve, qui s'impose et que Barthes diagnostiquait comme un degré zéro dès les années 1950. Notre époque semble ainsi avoir perdu depuis longtemps le sens de la verve. Avoir oublié la beauté de cette prosodie hors norme, la magnifique incantation que réalisent ces textes proliférants, ces phrases débordantes, le sentiment de quelque chose d'immédiat, de spontané, d'affectif, qui a malgré tout été longuement mûri, concerté, travaillé. Et pourtant, à considérer la trajectoire de tant d'écrivains, ce qui frappe c'est que la verve appartient souvent aux premiers temps de l'écriture, à son enfance révoltée, à ses indignations fondatrices. Que certains (Nizan, Le Clézio, Modiano) ont commencé leur œuvre par des éclats frondeurs, par une verve qui les a nourris, éduqués, puis apaisés, avant de s'éteindre. Mais beaucoup plus rares sont ceux qui, comme Albert Cohen, ont maintenu le cap de la verve d'un bout à l'autre de leur parcours. Car jamais l'auteur de Belle du Seigneur n'a choisi la voie de la tempérance. Jamais il ne s'est fatigué d'un ton inimitable. Mais il n'installa pas non plus sa verve dans une routine confortable. Intempestive, celle-ci ne perd jamais de sa force de séduction. Envahissante, elle s'empare de tous les sujets et court de la première à la dernière page de chaque texte. Parler de verve chez Cohen, c'est alors affronter une question délicate tant elle est partout présente, et tant elle articule le plan du récit comme énoncé et celui du récit comme énonciation. C'est évoquer un complexe réseau d'interactions entre la parole des personnages, celle du 
narrateur et une sorte de poétique de la lecture, voire une éthique de la littérature, qui se détermine dans et par cette verve.

\section{Du verbeux au verveux}

L'œuvre de Cohen, à la manière de celle de Proust, se caractérise par une attention aiguë à tous les phénomènes de langage. Elle s'oriente vers une forme de sociologie romanesque qui jamais n'épouse la logique paralysante du sérieux. Minutieux, le regard porté sur la parole est attentif aux sociolectes et aux idiolectes de chacun, les retranscrivant avec le plus grand soin, depuis les déformations lexicales ou syntaxiques jusqu'aux accents. Mais, parmi cette constellation polyphonique, parler pour ne rien dire, pour ne pas se faire entendre, s'adonner au brouhaha du verbe pour combler le défaut qui fore l'être et la pensée : tel est le trait définitoire d'une certaine verve propre au discours politique et diplomatique. Car à la SDN et chez les mondains, tous font des gorges chaudes de la moindre trouvaille linguistique. Chaque nouvelle habile tournure provoque le ravissement des fonctionnaires:

Les notes de van Vries faisaient l'admiration de ses collaborateurs. Il était en effet passé maître dans l'art de ne rien dire. Atteint de circonspection pathologique, ce fonctionnaire était capable d'aligner des douzaines de phrases paraissant pourvues de signification mais qui, relues attentivement, n'en avaient aucune et ne pouvaient donc engager sa responsabilité. C'était le talent imbécile de savoir ne rien dire en plusieurs pages.

Ergoter est en effet le sport favori de ces hommes qui s'ennuient : Le Gandec vient par exemple consulter Adrien pour savoir si «se ranger à notre opinion" est idoine ou si «se ranger à notre point de vue» ne serait pas plus adéquat ${ }^{2}$. Adrien, tout fat qu'il est, est comme un poisson dans l'eau. Il s'enorgueillit d'avoir eu l'idée d'«employer le mot développement au pluriel, ce qui lui donnait plus de gravité» ( $M$, p. 484) :

Et le plus haut fleuron de sa couronne, cette tournure ingénieuse grâce à laquelle, lorsqu'il était impossible d'éluder un "en ce qui concerne», on pouvait au moins en éviter la répétition. Autrefois, ses collègues écrivaient par exemple «tant en ce qui concerne la Syrie qu'en ce qui concerne la Palestine». C'était à lui qu'on devait le «tant en ce qui concerne la Syrie que la Palestine» $(M$, p. 484$)$.

C'est tout un idiolecte verbeux que Cohen passe en revue, où le signifiant prend le pas sur le signifié, où la syntaxe prime sur le réel. Cet univers s'inscrit

I. A. Cohen, Belle du Seigneur [1968], 1998, p. 338 (désormais BS).

2. A. Cohen, Mangeclous [1938], I980, p. 478 (désormais $M$ ). 
de facto dans une crise de l'éloquence et de la verve, signant une crise plus profonde de la pensée et de la communication, une anesthésie de notre rapport aux mots mais aussi aux choses et aux autres.

Il s'agit donc de saisir au plus près de la verve stéréotypée des mondains et du plaisir qu'ils y trouvent, des symptômes, des névroses, tout en sortant la langue qui les dit de sa routine et de ses marottes. Avec Cohen, il faudrait faire surgir l'extraordinaire de la verve depuis une parole verbeuse où la langue disparaît derrière sa fonction de plate communication, où elle s'enlise dans des formes évidées de tout sens. La véritable verve s'origine dans cette anfractuosité où un langage est ressuscité en l'étudiant et en le parodiant. Si analyse du discours, des idiolectes et des sociolectes il y a, celle-ci vaut donc aussi et surtout par son caractère passionnel et verveux. Elle n'existe que comme désir profond face à la verve, goût du jeu avec les mots en réponse au dégoût face au jeu stérile des mondains. Contrairement à Stendhal, grand modèle du romancier à tel point que Le Rouge et le Noir constitue l'un des intertextes majeurs de Solal, Cohen, tout en critiquant la verve aride des mondains, comme Stendhal celle des ecclésiastiques ou des politiques, n’a donc pas peur de recourir lui aussi à la verve. Or, si l'éloquence parfois contrefaite de Julien est mise à distance régulièrement, celle des Valeureux et de Solal n'est jamais dénoncée. Car elle est essentielle et vitale. C'est ainsi la parole virtuose des cousins de Céphalonie qui «ébahissait cette population de péroreurs orientaux ${ }^{3}$ » qui forme le petit peuple juif de l'île. Éloquents parmi les éloquents, les Valeureux forment l'aristocratie des verveux. Mais c'est certainement Mangeclous qui se présente comme l'orateur le plus forcené et le plus impénitent du groupe. Sa rhétorique de l'outrance est partout à l'œuvre, et les Céphaloniens écoutent avec plaisir ce hâbleur à qui ils offrent facilement des tribunes pour réaliser ses prêches désinvoltes et iconoclastes, comme l'université de Céphalonie dont il sera le recteur. C'est que cette verve recèle des possibilités insoupçonnées de dialogue avec l'autre devenu un véritable vis-à-vis, qu'elle témoigne d'un autre rapport au monde.

\section{Au commencement était l'excès}

La verve chez Cohen, qui passe autant par la parole de certains personnages, comme Mangeclous et Solal, que par celle du narrateur, a alors besoin de l'hyperbole et de l'incongruité parce que le réel est perçu par tous dans une logique euphémisante et stéréotypée qui l'affadit. Elle témoigne d'une tentative pour combler un vide au cœur du monde, une lacune ontologique,

3. A. Cohen, Les Valeureux [1969], 1986, p. 8I (désormais $V$ ). 
métaphysique et existentielle. Ni pointillisme ni laconisme avec cet écrivain qui a toujours la verve haute. Celle-ci n'a jamais le caractère plat et calme, minutieux, appliqué et sobre du bavardage ou de la badinerie. Elle est pleine de reliefs, de pics, de pointes, de chutes et de falaises. Loin de la prudence stendhalienne, le ton Cohen est un ton outrancier, qui passe toutes les mesures, qui joue de toutes les transgressions, souvent liées à la parodie ou au pastiche du discours amoureux, politique, littéraire, mondain, parfois même biblique. Il y a ainsi avec Cohen un certain art à la fois d'enchaîner le discours et de le couper net; bref de le rendre insensé. Car la verve est toujours court-circuit. Débordement.

Elle a donc trait à un plaisir de l'excès ne tenant pas aux lois usuelles de la communication, qui reposent notamment sur la progression du discours, mais à leur transgression. Car Cohen fonde la verve du texte dans un ressassement désarmant, qui est aussi surplace parce qu'il est le lieu de la jouissance qu'on n'arrive pas à quitter. La verve chez lui est en effet rarement brève, retenue, sous la forme de l'éclair. Elle s'étend au contraire, ne naît que de la démesure, parce qu'une fois mise en route elle ne peut plus s'arrêter. Les discours sur l'amour, qu'ils soient déclarations de passion avec Solal ou critique burlesque du romantisme avec Mangeclous, les descriptions de la mondanité, les lamentations des personnages, les dialogues des Valeureux, les monologues intérieurs : tous se caractérisent par une longueur exceptionnelle. Contrairement à Stendhal ou à Gide, Cohen ne pratique pas l'art des idées sautées, des ellipses et des litotes. Il se place dans l'art du trop-dire, de l'insistance. Non dans ce qui est ôté mais dans ce qui est surajouté. Non seulement en quantité (ce que le romancier appelle "la prolifération cancéreuse ${ }^{4}$ ) mais aussi en intensité. Lorsque la parole est en verve, c'est toujours dans l'extension, l'explosion presque. La phrase et l'idée reviennent alors en variantes et variations thématiques et formelles, comme si la formulation n'était jamais pleinement suffisante, jamais assez aiguisée, ne trouvait jamais sa forme définitive. La verve conjugue ainsi un principe de satisfaction à un principe d'insatisfaction. Le plaisir tient ici à la prouesse de tenir la distance, de se renouveler en se répétant, en étendant sans fin la scène, de ne jamais lasser. La gageure est de soutenir une verve quasi constante : de la maintenir en la variant, d'entretenir un lecteur, nécessairement intermittent dans ses joies, dans un état d'excitation quasi permanent. Car jamais Cohen ne s'installe définitivement dans un ton. Si une tonalité peut colorer un long passage, le romancier ne tient jamais la note mais joue d'une incessante polyphonie, de crescendo, glissando, parfois da capo ou sotto voce. C'est donc selon

4. "Tous mes livres ont été écrits par amour», Le Magazine littéraire, 1979, p. 7. Voir aussi "Je suis un archer qui tire dans le noir", Journal de Genève, 1969, p. 15. 
une rythmique, une prosodie, une pulsation, que se donne la verve et qu'elle se module. Il n'y a pas une verve mais des verves. C'est ainsi ce maintien paradoxal qui devient le liant d'un texte aux tonalités si disparates. La verve est l'unité secrète et la mesure démesurée des romans. Car il y a verve jusque là où elle s'infléchit, cesse, revient autrement, dans le malheur et la souffrance par exemple. Le plaisir nait ainsi non seulement de l'oscillation mais aussi de retrouver le même autrement, de revenir à une tonalité délaissée, à une verve qui fait retour alors qu'on l'avait presque oubliée.

Ce qu'on constate alors c'est combien la phrase de Cohen peut être vue comme un microcosme qui propose une image condensée de cette verve haletante. La syntaxe des phrases repose sur la subordination en chaîne, la coordination, la parataxe, l'épanorthose, la surcharge dans la caractérisation, notamment adjectivales. Elle est en elle-même une des formes de l'hyperbole. Profuse jusqu'à être ek-centrique, la phrase tient comme en équilibre. Pleine de propositions qui ne sont nullement des épines dorsales mais chaque fois de nouveaux foyers de décentrement, elle brise sans cesse sa propre structure par de nouveaux modes d'insertion d'éléments surabondants. La proposition principale n'y est donc jamais tout à fait principale : tout ce qui gravite autour d'elle parle, bavarde, grouille, s'anime, vit. C'est la cascade qui est le modèle de la phrase comme de la succession des phrases. Modèle dynamique mais aussi prosodique, harmonique et tonal. L'unité n'est donc jamais à rechercher en elle seule. Car la verve induit Cohen à écrire non par phrases mais par paquets de phrases, par grappes ou agrégats, par paragraphes presque, parfois même par chapitres entiers, comme c'est le cas avec les monologues intérieurs autonomes. Cette verve fait que chaque phrase renvoie à une autre, chaque paragraphe à un autre, chaque chapitre à un autre. Si bien que c'est le langage tout entier qui pourrait être caressé, peut-être même inclus dans le livre. Les nombreuses listes qui parcourent les textes, en particulier celles des métiers de Mangeclous ${ }^{6}$ ou de la qualité des pets de sa femme ( $M$, p. I33-I34), sont la forme débridée de ce fantasme encyclopédique où ce sont à la fois les mots et les choses qui s'accumulent et s'actualisent sous nos yeux. La verve est ainsi pressentiment d'une totalisation qui se donne seulement dans et par le langage. Elle est une manière de situer l'homme (le personnage mais aussi et surtout le lecteur) à l'intérieur d'un langage torrentiel.

5. Notamment dans des séries d'adjectifs accolés dont on ne peut plus dire quel est le lieu d'incidence réel. Un exemple entre mille : "la confuse honteuse conscience que nous avons tous de notre nature babouine» ( $B S$, p. 404).

6. A. Cohen, Solal [1930], I98I, p. I9-20 (désormais $S$ ). Ainsi que $M$, p. 48-49 et $V$, p. I5. 


\section{Il n'y a pas d'inconscient de la verve}

La verve n'est donc jamais quelque chose que le romancier cherche à masquer. Son travail sur l'éloquence, il ne tente pas de le polir mais au contraire le rend hautement visible, lève le voile sur ses soubassements, sans qu'il cesse de se donner pour naturel et spontané. Mangeclous a par exemple conscience d'être maître ès rhétorique, ce qu'il ne manque jamais de nous signaler :

C'est l'exorde, mise en train nécessaire et partie indispensable d'un discours; moelle de l'éloquence et structure fondamentale de l'art oratoire! ( $B S$, p. 72I)

Ayant ainsi résumé de manière sobre mes principaux arguments, je passe de la philippique à la péroraison, avec changement de registre et déviation vers la tendresse. (BS, p. 723)

Les enfants du fantasque rhéteur utilisent d'ailleurs la même terminologie en se référant à l'exorde, aux prolégomènes, à la harangue... ( $V$, p. 36) C’est dans cette perspective qu'il faut aussi comprendre une singularité frappante de la verve séductrice de Solal. Que ce soit devant Adrienne ( $S$, p. I3O-I34) ou devant Ariane ( $B S$, p. 385-439), le héros est incapable de reconduire l'argumentation éculée des don Juan qui l'ont précédé. Sa verve relève de tout autre chose : il s'agit de séduire en expliquant la manière dont on séduit, en exhibant une virtuosité démonstrative qui atomise la phraséologie éculée des Casanova, en assumant une verve réflexive qui se constitue uniquement à mesure qu'elle définit sa propre rhétorique. Là se tient tout un traité sur l'éloquence où l'effet de déshabillé linguistique, chargé d'érotisme et de mystère, sidère jusqu'au vertige ses destinataires et le lecteur. Avec Cohen, il n'y a donc pas d'inconscient de la verve. Car tous ces dispositifs métalinguistiques viennent régulièrement rappeler au lecteur que la parole a un poids, une épaisseur, une couleur, que les digressions restent sous contrôle, que le séducteur tient le gouvernail, que le fabulateur navigue sans jamais s'égarer, qu'un dispositif argumentatif très précis est toujours à l'œuvre, même souterrainement, dans la parole la plus libre possible. Ils rendent manifeste une tradition de l'éloquence que les Valeureux ou Solal maîtrisent d'un bout à l'autre sans qu'ils y découvrent des recettes toutes prêtes ou du pré-pensé, mais à laquelle ils parviennent à adosser leur propre créativité.

Or c'est à l'intérieur même de certains des procédés de cette verve que celleci s'avoue et se met à nu sous nos yeux fascinés. Si l'on examine la rhétorique valeureuse, on repère aisément un certain nombre de phénomènes stylistiques qui y concourent. Celle-ci organise un trouble permanent en hybridant les registres, en accouplant les termes archaïques, étrangers et les néologismes les 
plus surprenants, en tordant les us et coutumes du langage par une inventivité sans commune mesure dans la formulation des louanges ou des insultes ${ }^{7}$. Ce qui se met en place n'est autre qu'une zone de turbulence où une lecture passive, qui fait la bascule immédiate et instinctive du signifiant au signifié, n'est plus possible. Les périphrases descriptives, qui, augmentant le volume du discours, appartiennent à l'emphase et servent d'amorce à l'efflorescence de la verve, visent elles aussi à soustraire le langage à ses figements lexicaux qui masquent le réel alors qu'ils nous donnent le sentiment factice de toucher la vérité des choses ${ }^{8}$. Regardons par exemple ce qu'il advient d'un simple taxi et de son compteur, la manière dont ils peuvent susciter un nombre quasi infini de désignations qui se construisent comme des variations descriptives sur un thème unique : "carrosse mobile à vapeur intérieure, se mouvant seul mais payant et à horloge augmentante» (BS, p. 7I7), «horloge de l'abus» (p. 718), "voiture ambulante par sa propre volonté» (p. 722), "voiture des dilapidations» (p. 746), "horloge des débours» (p. 746), «horloge des francs helvétiques» (p. 752). Cet aliment de la verve qu'est la périphrase s'amuse du figement des expressions que nous employons usuellement, pour les rendre à une mouvance surprenante. Deux éléments constants reviennent. D’abord le déplacement autonome, qui dénote non seulement l'étrangeté des Valeureux par rapport à l'univers qu'ils décrivent, mais surtout leur rapport premier au mot, qui passe par leur étymologie (l'automobile est le véhicule qui se meut de lui-même). Ensuite la facturation mesurée à l'aune d'une durée. De ces deux éléments, presque obsessionnels, naît une combinatoire frappante qui introduit sans cesse l'affect et la labilité dans la désignation, obligeant le lecteur à sans cesse reconsidérer les objets du monde qu'il tient pour si coutumier que son regard ne s'y arrête plus.

Pour mesurer la diversité de ces processus, prenons une simple phrase de Mangeclous, extraite d'une vaste argumentation destinée à émouvoir Michaël qui refuse de livrer un secret sur Ariane et Solal :

Comptes-tu pour rien mon humiliation lorsque, reçus par le neveu de Saltiel à huit heures du soir seulement, nous lui communiquâmes la feintise hérö̈que de son oncle, à savoir qu'il était resté à Athènes, et qu'alors ledit neveu nous renvoya, moi, Mattathias et Salomon, t’accordant la préférence incroyable de te garder seul avec lui tandis que, paria et penaud à l'extrême et dévoré d'humiliation imméritée qui changea mon cœur en fange noire, je partis avec ceux-ci en grande contrition et perte de face attendre ton retour que nous espérions proche et fraternel dans l'hôtel démuni d'eau courante où nous t'attendîmes, non sans avoir au préalable

7. Une entre tant d'autres : «ô ongle du petit orteil, ou même sa rognure» (BS, p. 720).

8. Sur ce vaste sujet, on pourra par exemple consulter M. Murat, «La périphrase : remarques autour d'une figure", L'Information grammaticale, $\mathrm{n}^{\circ}$ 13, 1982, p. 38-40. 
fait l'emplette, à ton intention autant qu'à la mienne, de force boissons et délicieuses provisions de bouche chez cet épicier et traiteur israélite de Salonique qui reste ouvert jusqu'à minuit et dont $j$ 'achetai avec grandeur et largeur de paume, la dépense m’important peu car ma mort m'est toujours présente avec agonie préalable, aggravée de spasmes, étouffements, griffures de poitrine et râles divers, et je crache sur les pièces d'or, seriatim et privatim, dont j'achetai, dis-je, tout le stock de mets préparés, y compris même de charmants calmars tout juste arrivés de Marseille et qu'il fit frire à grande friture bruyante en ma présence! [...] Comptes-tu pour rien cette générosité $[\ldots] »(B S$, p. 720)

Phrase tentaculaire qui s'agite dans un pathos surjoué et comique, et qui heurte notre tranquillité par un jeu de trompe-l'œil où nous sommes invités à mener l'enquête pour identifier ou démasquer les expressions figées vraies ou fausses". Si le lecteur quelque peu érudit reconnaîtra les termes "provisions de bouche» comme une formule usitée, sa rareté empêchera pourtant de l'admettre spontanément car elle confine à l'incongruité. L'association de deux termes latins, peu fréquents en français, «seriatim et privatim», et plus souvent mobilisés dans la langue anglaise que les Valeureux prisent tout particulièrement, se présente de son côté comme le pastiche d'une locution figée. C'est ainsi la capacité qu'a Mangeclous d'inventer des expressions qui se donnent comme des idiotismes aberrants qui est manifeste. Ainsi en va-t-il de l'emploi surprenant de "perte de face", syntagme inexistant dans la langue mais résultant de la substantivation de la locution verbale "perdre la face». C'est ici le transfert catégoriel qui assure le défigement de l'expression. Le tour «avec grandeur et largeur de paume» relève d'un fonctionnement sensiblement proche. Là encore il s'affiche comme un syntagme figé, forgé de toute pièce et qui se construit par mimétisme sémantique sur l'expression «avoir le cœur sur la main». La paume est ici prise comme une métonymie de «main», qui, dans l'expression source, était métonymie de la générosité et du don, sèmes qui sont actualisés dans les noms "grandeur et largeur» qui doivent être interprétés non dans leur sens propre et physique mais dans leur sens figuré. Cette accumulation de déplacements et d'extensions est un tour particulièrement habile pour inclure la réflexivité métalinguistique au sein de la parole. Elle présuppose en effet une série d'opérations mentales relativement longue de la part du lecteur qui, dans la rapidité du discours verveux, contraint en permanence à faire retour sur le langage lui-même, à conserver une conscience du discours et de son fonctionnement.

La verve présuppose ainsi chez Cohen l'emploi d'une langue volontairement idiolectale et minoritaire qui se donne non comme dominatrice mais

9. Sur la notion d'expression figée, on renverra à G. Gross, Les Expressions figées en français. Les noms composés et autres locutions, 1996. 
comme exemplaire. Se jouant de tous les figements hiératiques, elle indique elle-même qu'elle n'est jamais un état stable qu'il conviendrait d'imiter ou d'imposer, mais qu'elle est toujours en cours de dépassement, d'abolition et de recréation. C'est son ouverture à toutes les paroles qui s'exhibe, son caractère plastique et généreux. Non assertive, la verve se pense comme oblative. Elle met en acte un dérèglement du monde qui s'appuie sur une subjectivité qui voudrait être partagée, comme dans le rêve d'une collectivité à venir dont le groupuscule des Valeureux, uni par une verve communicative et contagieuse, serait une sorte d'exemplum à suivre.

\section{Et Verbum caro factum est}

Mais si la verve est constante dans l'écriture, elle a ses sujets de prédilections qui déterminent son expression. Il lui faut une impulsion initiale. Un combustible de choix. Une étincelle qui l'allume. La passion, le social, la force, les totalitarismes, l'antisémitisme, la judéité, le corps sont ses thématiques de choix. Subsumante, elle s'exprime indépendamment des différents styles qu'elle peut être amenée à mobiliser, et s'apparente à une sorte de ton, une couleur du discours qui se passe finalement de stylèmes caractéristiques. Tantôt lyrique, polémique, comique, triviale, la verve est mouvement, dynamique, flux. Chevillée à une sorte d'accroche, de point de départ qui la meut et l'é-meut, elle est envol d'une voix, comme dans la logique du lyrisme, décision de parler, marque d'un idiolecte ${ }^{\mathrm{I}}$. Il n'est pas étonnant que la plupart des discours verveux, qu'il s'agisse de ceux du narrateur, de Solal ou des Valeureux, recourent si volontiers à l'apostrophe "ô ", même en dehors de toute tonalité lyrique. On la rencontrera aussi bien lorsque le texte est une charge, une satire, une parodie, une réjouissance burlesque qu'un hymne à la passion. Marque par excellence

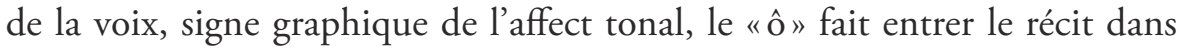
l'ordre du discours et nous donne à entendre une présence, celle de l'âme qui prend corps dans la verve. Il marque un point de départ et un aboutissement, il est la trace d'un enchaînement et d'une relance. Car ce qui pourrait devenir cri ou particule isolée, trouve dans le reste du texte le support de son énergie, y imprime la vibration de son élévation, se donne comme l'impulsion mais aussi la cristallisation en un point unique d'une verve à l'extension presque infinie. Endroit où la syntaxe n'existe plus, zone de turbulence autour de laquelle la verve se défait et se refait, l'apostrophe tend le langage vers l'autre,

IO. Dans cette perspective, on pourra lire avec profit les analyses sur l'apostrophe et l'exclamation dans le discours lyrique, de J.-M. Maulpoix, Du lyrisme, 2000, p. 225 et suiv. 
dans une sorte de court-circuit du contenu au profit d'un lien intangible et indescriptible. Elle est ouverture d'un espace de dialogue avec autrui, avec le monde et avec le désir qui porte la verve.

De l'ordre du souffle, la verve est donc aussi pneumatique. Elle a trait à l'oralité, celle du conteur qu'est le narrateur ou celle des orateurs que sont Mangeclous ou Solal. Elle a quelque chose à voir avec le ton, le timbre, le débit, le rythme. Au lecteur de Cohen, est ainsi donné à entendre, plus qu'à voir, un mouvement vibratoire et phonique de la parole qui n'est pas indifférent au phénomène émotif qui se constitue à la lecture. C'est donc bien aussi dans le rapport du texte à la voix, que la verve se constitue et accède à sa densité pleine. Si elle se donne comme une rhétorique, avec son inventio et sa dispositio, c'est surtout l'elocutio et l'actio qui priment. Elle relève de la performance.

Et c'est dans cet orbe que s'inscrit la si surprenante présence de la figure auctoriale que se veut le narrateur. Car Cohen, comme Stendhal avant lui, entrecoupe sa verve de métalepses qui s'affichent comme des interventions d'auteur ${ }^{\text {II }}$. Oscillant entre la jubilation exhibée ("Quant à Saltiel, il était plus blanc que la feuille sur laquelle j'écris avec un plaisir extrême car en ce moment j'entends une chanson populaire espagnole» $[M$, p. 257]) et la lassitude devant le verbe ("Je n’ai pas le temps de raconter les péripéties» [p. 264]), «Assez. On ne peut pas tout raconter, on n'en finirait plus» $[V$, p. 248]), elles disent surtout la nécessité pour le conteur de parler à son propre personnage :

Fils de mon cœur, petit Salomon, [...] petit mais vrai sauveur, il n'y a que moi qui t'estime et te respecte. Et tu es trop vrai grand humain pour le savoir, ô escargot, ô microbe, ô grande âme. Laisse-les sourire et se moquer de toi et va gambader, petit, tout petit immortel. Va, mon agneau, mon mignon messie chérir ( $M$, p. II3).

Ce commerce singulier, oralisé, individualisé, entretenu entre le personnage et la figure présumée de l'écrivain, passe par-dessus les frontières de l'univers du texte et du lecteur. Le narrateur affirme ainsi une sorte d'interdépendance avec certains personnages qui ne comptent pourtant pas parmi les plus verveux (Mme Sarles, Hippolyte, Jérémie, Salomon...). Plus que comédie de masques et d'artifices, ce jeu repose sur une empathie marquée qui vient interrompre le flot du texte où la verve s'épand comme une coulée de lave. Mais paradoxalement, cette suspension momentanée de la verve, ne sert qu’à la relancer sans jamais déconstruire la figure d'un narrateur-auteur particulièrement loquace. D'autant que, par ce rapprochement avec la temporalité du lecteur, la verve

II. Voir C. Stoltz, La Polyphonie dans Belle du Seigneur d'Albert Cohen, I998, p. 298-316. Extrêmement nombreuses, les métalepses sont parfois très ostensibles, parfois plus ambiguës et l'on ne peut pas toujours savoir si c'est la voix du personnage qui parle ou celle de l'auteur. Voir par exemple $S$, p. II5, 242, 245, 292, 377. 
affirme son caractère contagieux et généreux, elle nous rend contemporain de l'intrigue en incarnant (le mot doit prendre ici son sens le plus fort), la verve en un individu. La métalepse, cette solution de continuité de la verve, nous pourrions alors la comprendre comme un analogon littérarisé de cette parole fondatrice de saint Jean : Et Verbum caro factum est. Car c'est bien ainsi que Cohen interdit à sa verve d'être pure machinerie sans âme, rhétorique automatique. Au contraire, il lui insuffle quelque chose d'une anima, il lui donne vie et corps. La verve n'aurait donc de sens qu'à être ainsi soutenue par un être, à se faire incarnation d'une individualité.

Cette propension à la contagion et à hypostasier le verbe dans la personne, sans jamais se livrer à une logique stérile d'imitation comme dans l'univers mondain, mue la verve cohénienne en une entité diverse mais toujours subjective. À chaque fois, ce sont des sentiments différents qui l'animent mais qui, tous, puisent à un vaste nuancier où la colère, l'indignation, l'amour, le plaisir, l'étonnement, la jubilation se donnent la réplique. Elle n'est donc jamais dénuée de partis pris. Elle refuse d'être neutre. Elle affiche sa nature partisane, son axiologie, ses préférences, ses inclinations, et c'est ce qui fait son charme. Mais s'il est un sentiment qui lui est inconnu, c'est assurément la haine, le ressentiment. Car la loi du grief est toujours portée par une vitalité comique qui l'empêche d'être négation gratuite ou nihilisme péremptoire. C'est pourquoi la verve va au-delà de la simple rhétorique brillante. Si elle jette parfois un peu de poudre aux yeux, elle revêt un caractère éthique où la forme fait sens par elle-même et n'est pas que le simple véhicule ou le tuteur d'une pensée ou d'une idée. Elle est elle-même idée. Pas seulement sa propre idée mais idée de son contenu. C'est elle qui fait sens, qui vaut pour une attitude existentielle face au monde car est elle une manière de se positionner dans la parole par rapport au signifié.

Et c'est de la sorte qu'elle indique aussi la conception de ce que doit être la littérature en regard des idées qui y sont formulées. Un discours particulièrement verveux de Mangeclous, réécrivant les amours de "l'Anna» $(M$, p. 138) et de «l'amant, prince Wronksy et poète», met en abyme ce fonctionnement du texte, qui vaut à toutes les échelles (le mot, la phrase, le paragraphe, le chapitre, le roman), en dénonçant la continence des amants de la littérature. Après avoir évoqué la colique dudit prince, habilement dissimulée sous des serments énamourés et des vers clamés haut et fort, Mangeclous affirme qu'il est temps que cesse enfin cette censure du réel exercée par les romanciers de la passion :

Oui, messieurs, depuis Homère jusqu'à Tolstoï, les jeunes héros et héroïnes souffrent, surtout s'ils sont beaux, d'une épouvantable rétention. Ils n'en peuvent plus. [...] Tous les amants, toutes les amantes de Shakespeare, de Racine, de Dante n'en peuvent plus de la continence qui leur a été imposée par leurs auteurs. Ils 
se tordent de douleur, entrecroisent leurs jambes depuis des siècles pour rester convenables! Mais aujourd'hui, c'est la libération et la révolte! Moi, Mangeclous, je vous donne licence et permission, ô charmantes héroïnes et nobles héros de passion! Avouez que vous n'en pouvez plus! Vous tous, martyrisés du roman, finissez-en avec cette sécheresse et jaillissez enfin loin et fort, en un jet unanime et joyeux et véridique, franchement et fraternellement! Messieurs, j'ai fini ma péroraison! ( $M$, p. I4I)

Au-delà de l'allusion filée à la scatologie, ce morceau de bravoure plaide pour une libération de la parole, « un jet unanime et joyeux et véridique» qui, sur le modèle de la diarrhée, définit la verve comme une logorrhée. C'est, en dernière instance, la forme certes triviale mais essentielle d'une incarnation idiolectale de la verve dans ce qui l'a soutenu et défini si précieusement au cours des siècles avant de l'abandonner : la littérature.

\section{Bibliographie}

Cohen Albert, Solal [1930], Paris, Gallimard, coll. «Folio», I98I.

—, Mangeclous [1938], Paris, Gallimard, coll. «Folio», I980.

—, Belle du Seigneur [1968], Paris, Gallimard, coll. «Folio», 1998.

—, Les Valeureux [1969], Paris, Gallimard, coll. «Folio», I986.

—, "Tous mes livres ont été écrits par amour», entretien avec J.-J. Brochier et G. Valbert, Le Magazine littéraire, $\mathrm{n}^{\circ}$ 174, avril 1979.

—, "Je suis un archer qui tire dans le noir", entretien avec J. Buenzod, Journal de Genève, 20-2I décembre 1969.

Gross Gaston, Les Expressions figées en français. Les noms composés et autres locutions, Paris, Ophrys, 1996.

Maulpoix Jean-Michel, Du lyrisme, Paris, José Corti, 2000.

Murat Michel, «La périphrase : remarques autour d'une figure», L'Information Grammaticale, $\mathrm{n}^{\circ}$ 13, 1982, p. 38-40.

Stolz Claire, La Polyphonie dans Belle du Seigneur d'Albert Cohen. Pour une approche sémio-stylistique, Paris, Champion, 1998. 